\title{
Balkanologie
}

Balkanologie Revue d'études pluridisciplinaires

Vol. IV, $n^{\circ} 2 \mid 2000$

Volume IV Numéro 2

\section{Par delà le clivage est / ouest : l'expérience des terrains croisés}

Adina Ionescu-Muscel et Stéphanie Mahieu

\section{(2) OpenEdition}

1 Journals

Édition électronique

URL : http://journals.openedition.org/balkanologie/342

DOI : $10.4000 /$ balkanologie.342

ISSN : 1965-0582

Éditeur

Association française d'études sur les Balkans (Afebalk)

Édition imprimée

Date de publication : 1 décembre 2000

ISSN : 1279-7952

Référence électronique

Adina Ionescu-Muscel et Stéphanie Mahieu, «Par delà le clivage est / ouest : l'expérience des terrains croisés », Balkanologie [En ligne], Vol. IV, n² | 2000, mis en ligne le 22 avril 2008, consulté le 17 décembre 2020. URL : http://journals.openedition.org/balkanologie/342 ; DOI : https://doi.org/ 10.4000/balkanologie.342

Ce document a été généré automatiquement le 17 décembre 2020.

(c) Tous droits réservés 


\title{
Par delà le clivage est / ouest : l'expérience des terrains croisés
}

\author{
Adina Ionescu-Muscel et Stéphanie Mahieu
}

1 En 1994, la Société des Européanistes a eu l'initiative de créer une "Section Jeunes » destinée aux étudiants et jeunes chercheurs en anthropologie de l'Europe. Dans ce cadre a été mis sur pied le projet de « Terrains croisés », dont cinq éditions ont déjà eu lieu. L'idée initiale d'un tel projet était de permettre l'acquisition d'une première expérience de terrain - bien souvent absente des programmes des différentes universités européennes - dans un esprit de réciprocité, en bénéficiant de l'encadrement scientifique de membres de la Société des Européanistes ${ }^{1}$. À partir d'une telle expérience concrète, nous souhaiterions ${ }^{2}$ proposer une analyse plus générale, relative aux frontières qui sépareraient l'Est et l'Ouest de l'Europe, afin de nuancer l'hypothèse largement répandue d'une altérité radicale entre les « deux Europe ».

\section{Les « Terrains croisés » : premier bilan}

2 Quelques précisions s'imposent tout d'abord à propos de l'historique du projet des «Terrains croisés». Une expérience pilote a eu lieu dans le village de Treignes (Belgique) en août 1995 et a réuni des participants belges, italiens et roumains. C'est en juillet 1996 que le projet a véritablement pris son envol. Une vingtaine de participants roumains, belges et italiens ont passé un mois dans le village de Cristian (Roumanie), village dont l'ancienne population saxonne, récemment émigrée en Allemagne, a été remplacée par des populations roumaines et tziganes. Les enquêtes, menées autour de la question du départ des Saxons et de la réappropriation de l'espace physique et social par les nouveaux habitants du village, ont fait l'objet d'une publication, Cahiers de Terrain $1996^{3}$.

3 L'expérience de Cristian fut particulièrement enrichissante pour tous les participants, et a mené à la décision de mettre sur pied un projet pédagogique de longue durée, qui s'est concrétisé depuis lors par l'organisation annuelle d'un stage de terrain. Le stage de 1997 s'est déroulé à Salina, dans l'archipel des Éoliennes (Italie), celui de 1998 dans la 
région de Razgrad-Zlatia (Bulgarie), et celui de 1999 dans la ville de Petrosani (vallée du Jiu, Roumanie). Chacun de ces stages a réuni une trentaine de participants (étudiants et enseignants) venus de Belgique, Bulgarie, France, Grèce, Italie et Roumanie ${ }^{4}$, et a été organisé selon les mêmes modalités: les participants sont répartis en groupes « mixtes » comportant au minimum un étudiant du pays d'accueil, qui prend en charge la traduction des enquêtes et la transcription des entretiens. Cette particularité constitue un des fondements du projet des "Terrains croisés »: les participants « autochtones » ayant fait office de traducteurs une année, se verront, l'année suivante, placés de « l'autre côté du miroir ». Par ailleurs, le français a été choisi comme langue de travail et de rédaction du texte final que chaque groupe doit rédiger au terme du stage, et qui fait l'objet d'une publication.

4 Les stages de "Terrains croisés » ont fait apparaître le besoin des étudiants de bénéficier d'un cadre didactique leur permettant de mettre en pratique leur enseignement académique, même si une telle expérience ne s'inscrit pas à la ligne dans l'esprit «malinowskien » du terrain. Comme l'écrit Bojan Baskar,

il peut sembler contradictoire de plaider pour qu'une formation au travail de terrain soit introduite dans le cursus universitaire tout en postulant que la croyance dans les capacités particulières d'observation de l'anthropologue s'appuie sur le mythe malinowskien du terrain. Il n'y a cependant là aucune contradiction, si la recherche de terrain n'est pas jugée selon les critères du moule malinowskien. Les terrains de longue durée et l'observation participante sont sans aucun doute des éléments importants du modèle malinowskien, mais ils peuvent tout aussi bien en être extraits et utilisés dans le cadre d'autres dispositifs de recherche'5.

5 Le projet des «Terrains croisés ", dont l'objectif est avant tout pédagogique, présente de surcroît une double caractéristique. D'une part, chaque terrain permet le "croisement de regards", par la présence simultanée d'anthropologues «autochtones » et étrangers, issus des pays de l'Est et de l'Ouest de l'Europe. D'autre part, la possibilité qui est donnée aux étudiants de participer à plusieurs terrains successifs permet d'envisager un tel «croisement de regards » comme une relation fondatrice d'une optique multipolaire par rapport au terrain. Nous avons décelé dans une telle démarche un double décentrement, horizontal (ou synchronique), et vertical (ou diachronique). Au niveau horizontal, l'exigence d'un texte final commun, l'échange permanent de points de vue, les négociations entre les participants, relativise le statut des deux anthropologues (l'étranger et le local). Au niveau vertical, l'inversion des rôles que chacun jouera - hôte «accueillant » et hôte "accueilli »" - permet à la même personne d'expérimenter successivement les deux positions de sujet et d'objet ${ }^{7}$.

Dans une telle optique, les «terrains croisés » proposent aux étudiants d'acquérir tout d'abord les bases méthodologiques en vue d'apprendre à soulever et traiter une problématique ${ }^{8}$, à effectuer des entretiens, des traductions et des transcriptions et à rédiger « à chaud » un texte de systématisation. Toutes ces activités sont effectuées en groupes multinationaux, en gardant le souci constant de la mise en contexte des données recueillies et de l'interprétation qui est donnée à celles-ci. Par ailleurs, la présence de professeurs et d'assistants permet aux participants de bénéficier d'un encadrement scientifique prenant en compte leurs réactions face au terrain. 


\section{L' Europe des clivages?}

7 La disparition du rideau de fer s'est accompagnée d'une double conséquence pour l'anthropologie de l'Europe : «l'anthropologisation de l'ethnologie » (selon l'expression de Bojan Baskar) dans les anciennes républiques socialistes, et la plus grande mobilité des chercheurs aussi bien vers l'Est que vers l'Ouest. Le projet des "Terrains croisés » découle directement de ce nouveau contexte politique, dont une des conséquences est un intérêt anthropologique accru pour les relations entre l'Est et l'Ouest européen'.

Ces nouvelles préoccupations s'ajoutent aux anciennes interrogations qui ont conduit à l'émergence de l'anthropologie de l'Europe : le réinvestissement des terrains locaux et la critique de l'approche folklorique. Comme l'écrit Marianne Mesnil à ce sujet,

l'ethnologie européenne, par l'un de ces paradoxes (néanmoins explicable) que nous réserve l'histoire, est sans doute la dernière née des ethnologies régionales, puisqu'elle n'a pu constituer son objet et ses méthodes qu'à travers un double mouvement mené simultanément par rapport au folklore et à l'anthropologie. Procédant du premier, elle ne pouvait cependant émerger dans le champ des sciences humaines qu'au prix d'une rupture radicale mettant fin à la fusion (et confusion) qui s'était produite au XIXe siècle, entre "sujets des énonciations" (celles des folkloristes et nationalistes) et "sujets des énoncés" (le peuple producteur $\mathrm{du}$ "folklore" dont ils se faisaient les porte-parole). Ces discours apparurent d'ailleurs comme les enjeux de stratégies politiques, parmi lesquelles les revendications d'indépendance nationale ne furent pas les moindres. C'est à la condition d'une telle rupture qu'il devint donc possible de redéfinir des objectifs "à bonne distance" et de rejoindre ainsi les préoccupations et méthodes d'une anthropologie générale. Il faut par ailleurs souligner que la rencontre avec cette dernière discipline s'inscrit dans un contexte historique précis : celui, vers les années 1960-1970, où se manifeste la crise de décolonisation de ses terrains (...). Ainsi s'ouvrent de nouveaux champs d'investigations, "terrains à ethnologiser" à portée de la main ${ }^{10}$.

Le projet des "Terrains croisés» est conçu dans l'esprit d'une anthropologie de l'Europe telle qu'elle vient d'être définie. L'idée initiale qui a présidé à sa création était celle de la réciprocité qui devait permettre de dépasser une différence présumée entre les participants de l'Est et de l'Ouest. Mais lors des divers stages, nous avons été amenées à nous interroger sur la nature d'une telle différence. Au cours des dernières années, de nombreux auteurs, dont Samuel Huntington et Henri Mendras ${ }^{11}$, ont défini les frontières orientales de l'Europe, qui correspondraient soit au Rideau de Fer (Mendras), soit à la frontière politico-religieuse qui sépare les chrétientés occidentales et orientales (Huntington). Les pays situés à l'est de ces frontières constitueraient « l'Autre Europe ». L'utilisation d'une telle appellation prête à confusion : comme l'écrit Predrag Matvejevitch, «qu'est-ce qui est autre dans cette partie de l'Europe, qu'est-ce qui est européen dans cette altérité ? $»^{12}$. De plus, le terme d'« Autre Europe », avec tous les sous-entendus qu'il contient, est bien souvent conçu en terme d'opposition exclusive, qui renvoie les pays d'Europe centrale et orientale dans la sphère de l'altérité radicale.

10 Sans tomber dans l'attitude opposée qui consisterait à nier toute différence entre ces régions, il nous semble néanmoins nécessaire de nuancer le caractère définitif des clivages ainsi définis. Comme le précise Marianne Mesnil à ce sujet,

la conception d'une dichotomie "Orient/ Occident" n'a pas fini de puiser ses arguments aux sources les plus diverses, partout où l'on peut relever et accentuer des facteurs d'opposition (plus que de distinction) entre deux "cultures". Cette 
accentuation des différences qui fondent l'altérité, jusqu'à en faire des différends qui fondent l'hostilité, érigent d'une manière générale la "question identitaire" en problème ${ }^{13}$. la prise en compte des « différences / différends » lié à la présence de participants issus de six pays d'Europe de l'Ouest et de l'Est. Toutefois, il nous est apparu que la grille de lecture "culturelle», qui situe les «deux Europe» dans des mondes opposés, ne suffisait pas à rendre compte des difficultés qui se sont présentées lors des stages. Nous proposons donc l'hypothèse que ces difficultés sont d'ordre méthodologique plutôt que culturel, en précisant néanmoins que nous ne considérons pas le registre méthodologique comme indépendant du contexte historique, social, et politique dans lequel la discipline anthropologique évolue. De plus, tout en étant conscientes de l'importance de la prise en compte des particularités d'ordre individuel pour une analyse de la dynamique relationnelle qui s'instaure sur le terrain, nous nous limiterons ici au seul aspect intellectuel d'une telle expérience. pays, l'enseignement de l'anthropologie de l'Europe recouvre une très grande disparité de contenus, largement influencés par le triple héritage ethnologique, sociologique et folklorique. Ce problème, qui est plus significatif qu'une seule différence d'appellations, est lié à l'histoire de l'anthropologie dans chacun des pays participants aux « Terrains croisés $»^{14}$. Chacun des stages a été le théâtre de conflits, tensions et négociations, chacun étant persuadé de la justesse de «sa façon» de faire de l'anthropologie: les anthropologues à formation « classique » en vertu de l'approche comparative associée à leur discipline, les anthropologues à formation ethnographique grâce à leur expérience de terrain et les anthropo-sociologues de par leur approche quantitative bien rodée. Ces tensions se doublaient d'un enjeu plus implicite, celui de la quête du regard le plus juste, les "locaux » étant érigés en "experts culturels", alors que les étrangers étaient les détenteurs du « regard éloigné ».

13 Lors des différents stages de terrain, nous avons donc été confrontés à des difficultés d'ordre essentiellement communicationnel, et nous sommes arrivées à la conclusion que ces difficultés relèvent plutôt d'approches méthodologiques différentes, induites par un parcours différent de la discipline anthropologique dans chaque pays, que d'un clivage culturel Est / Ouest. En effet, il est apparu qu'il était parfois plus difficile de se mettre d'accord sur la façon de faire de l'anthropologie entre deux Italiens ou deux Bulgares issus de facultés différentes qu'entre un Français et un Roumain. Même si cet exemple peut paraitre banal, il soulève néanmoins la question essentielle de l'interprétation qui est faite des données récoltées. Cette question intervient beaucoup plus dans l'apparition des « différences / différends » entre les divers participants que leur appartenance culturelle. Comme l'écrit Bojan Baskar,

le partage réel se fait entre ceux qui savent que faire de l'ethnographie et ceux qui ne savent pas. L'anthropologue en pantoufles, comme Marcel Mauss, ou celui dont le travail de terrain ne correspond pas aux canons du modèle malinowskien, comme Lévi-Strauss, n'est pas une exception à la règle d'or selon laquelle le terrain malinowskien ferait les anthropologues : il est sur le même plan que Malinowski, sur la même rive. De l'autre côté de la ligne de partage, il y a le "collectionneur de papillons" dont l'ethnographie n'est pas destinée à être utilisée, n'ayant d'autre but que d'être collectée, et le "cerveau" auquel sa fureur théoricienne interdit la lecture du moindre livre ethnographique. ${ }^{15}$

Balkanologie, Vol. IV, $n^{\circ} 2$ | 2000 
$14 \mathrm{Au}$ cours de l'exercice "croisé » qu'a constitué l'écriture de cet article, nous avons tenté d'avancer une première lecture anthropologique d'une expérience qui nous a fortement marquées. L'enjeu qui apparaît au-delà de notre propos concerne la difficulté de rendre compte de la différence, en évitant les deux pièges symétriques que constituent son accentuation et son déni. Une telle démarche, qui a pour ambition de sortir de la logique identitaire ${ }^{16}$, implique l'élaboration d'un outillage conceptuel qui envisage les frontières et les appartenances collectives en termes non-exclusifs.

\section{NOTES}

1. Nous tenons à exprimer notre gratitude envers Marianne Mesnil et Vintila Mihailescu qui, par leur enthousiasme, leurs encouragements et leur encadrement scientifique, ont permis au projet de "terrains croisés" de se concrétiser et de se développer. Le croisement de regards que nous tentons d'illustrer par le présent article est né d'une démarche comparative qu'ils ont initiée. Nos remerciements vont également à Ilia Iliev, Rajna Gavrilova, Assia Popova, Ivailo Ditchev.

2. Le texte qui suit tente de refléter une partie des discussions menées entre les participants au cours des cinq expériences des "Terrains croisés". Néanmoins, la grille de lecture proposée n'engage que les auteurs de ces lignes.

3. Cahiers de Terrain 1996, Bucarest, Editions Paideia, 1997, 61 pages (recueil de 6 articles), publié par la Société des Européanistes et l'observatoire social de Bucarest ; Cahiers de Terrain 1998, Sofia, Editions LIK, 1999, 95 pages (recueil de 11 articles), publié par la Société des Européanistes et l'Université de Sofia.

4. Les universités participant au réseau des “Terrains croisés" sont: l'Université Libre de Bruxelles, Université Saint Clément d'Ohrid de Sofia, Université Lyon II-Lumière, Université Aristote de Thessalonique, Università La Sapienza de Rome, Università degli Studi di Cagliari, Università degli Studi di Perugia, Universitatea Bucuresti, Universitatea Babes-Bolyai Cluj.

5. Baskar (Bojan), «L'anthropologie sociale dans l'“autre Europe". Incertitudes et désagréments », Terrain, (31), septembre 1998, p. 127.

6. La langue française utilise le même terme d'hôte pour désigner celui qui accueille et celui qui est accueilli. Sur l'ambivalence du doublet étymologique hospes / hostis, voir Mesnil (M.), Mihailescu (V.), « La table sens dessus dessous. De l'hospitalité agonistique », Revue du M.A.U.S.S., (12), 2e semestre 1998.

7. Dans De l'angoisse à la méthode, G. Devereux, a jeté les bases d'une prise en compte de la distance infranchissable qui existe dans les sciences humaines entre l'objet et le sujet, en s'inspirant de la théorie freudienne du transfert/contre-transfert, in Devereux (Georges), De l'angoisse à la méthode dans les sciences du comportement", Paris : Aubier, 1980 (première édition 1967). Une telle grille de lecture nous semble pouvoir rendre compte de l'expérience partagée par les participants aux stages. En effet, de par son rôle "d'expert culturel" pour l'anthropologue étranger, l'anthropologue local est visé en tant qu'objet d'étude, dans la mesure où c'est à travers lui que se fait le décodage culturel. Par ailleurs, son association avec l'anthropologue étranger dans un projet commun lui confère le rôle de sujet de la recherche. Une telle dynamique se retrouve inversée l'année suivante, quand "l'hôte accueillant" devient à son tour "l'hôte accueilli". 
8. Précisons toutefois qu'un balisage préalable est effectué, afin de présenter le contexte général dans lequel s'inscrit la recherche.

9. Cet intérêt existait cependant avant la chute du mur, mais de manière beaucoup moins généralisée. Voir à ce sujet les travaux de J. Cuisenier, J.-F. Gossiaux, C. Hann, G. Kligman, M. Mesnil, K. Verdery, etc.

10. Mesnil (Marianne), «Perspectives de la recherche en ethnologie européenne », Civilisations, XXXIV (3-4), 1984, pp. 155-162.

11. Huntington (Samuel), Le choc des civilisations, Paris : Odile Jacob, 1997 (édition anglaise 1996); Mendras (Henri), L'Europe des Européens. Sociologie de l'Europe occidentale, Paris : Gallimard, 1997.

12. Matvejevitch (Predrag), Le monde "ex". Confessions, Paris : Fayard, 1996, p. 49.

13. Mesnil (Marianne), « Kosovo : les bombes à retardement d'un modèle occidental dans les Balkans », L'Écho, 5 mai 1999.

14. Une telle disparité se reflète dans les facultés très diverses dont sont issus les participants aux stages: faculté de sciences sociales, faculté de philosophie et lettres (section histoire et archéologie), faculté de psychologie, faculté de culturologie, faculté d'ethnologie, voire même faculté vétérinaire! Chacune de ces facultés délivre néanmoins l'équivalent d'un diplôme en anthropologie de l'Europe.

15. Baskar (Bojan), art. cit., p. 128.

16. Pour une critique de la logique identitaire, voir notamment Laplantine (François), Je, Nous et les autres, Paris : Editions Le Pommier, 1999.

\section{AUTEURS}

\section{STÉPHANIE MAHIEU}

Centre de recherches en ethnologie européenne, Université Libre de Bruxelles 